\title{
Tratamiento de la Vejiga Hipertónica e Hiperactiva en la Infancia con Sulfato de N-Metil Hioscina
}

\author{
Dr. Fingenio Rodriguez S. 1 ; Dr. Jojge Holzer M. 2; Dra. Ximena Triviño B. ${ }^{3}$; \\ Dr. Carlos Saieh A.1; Dr. Federico Puga C. 1 \\ Treatment of Patients with Hyperreflexive and Hypertonic \\ Bladder with N-Methylhioscine Sulfate
}

\begin{abstract}
Twenty one children with hyperreflexic bladder and 9 withlypertonicbladder were submited to urodinamic and clinical studies. Al were treated with $\mathrm{N}$-Methyl hioscine sulfate during 90 days and urodinamic evaluation was repeated at the end of the treatment period. Bladder capacity and post voiding residual volume increased, but no statistically significant growth was found in the bladder. Disappearance and shortening of the hyperreftexic segment and diminution of non inhibited contractions of the bladder were observed. The patients with hyperreflexic bladder showed elinical improvement of enuresis, encopresis and binder incontinence: this was unrclated with their urodinamic state at the end of the study. In contrast, patients with hypertonic bladder showed no imptovement in enurctic symptoms. Lndesirable side effects were not noted in this experience.

(Key words: N-Methythioscine sulfate. Enuresis. Bladdor incontinence).
\end{abstract}

En trabajos anteriores demostramos que los trastornos vesicales funcionales más frecuentes en la infancia son la vejiga hipertónica y la hiperactiva, que se manifiestan por infección urinaria recurrente, enuresis, incontinencia y encopresis, en forma asociada o aisladal.2.

1. Unidad de Nefrología, Hospital Luis Calvo Mackenna.

2. Instituto de Neutocirug ía e Investiguciones Cerebrales.

3. Becada de Pediatria, Hospital Luis Calvo Mackenna.
Es conocido el efecto benefìcioso de algunos fármacos anticolinérgicos sobre estas disfunciones vesicales, al disminuir la actividad de la musculatura lisa del detrusor $3,4,5,6,7$.

El objetivo de este trabajo es evaluar la res. puesta clínica y cistométrica de pacientes con vejigas hiperactivas e hipertónicas sometidos al tratamiento con sulfato de $\mathrm{N}$-Metil Hioscina (ULIX®), Este fámaco fue escogido por sus propiedades anticolinérgicas, baja toxicidad, disponibilidad en el mercado nacional como monodroga y costo relativamente bajo ${ }^{3}$. 


\section{MATERIAL Y METODO}

Entre Junio de 1984 y Junio de 1985 se esiudiaron 30 nitios controlados en la Unidad de Nefrourología del Hospital Luis Calvo Mackenna por infección urinaria recurrente, enuresis, incontinencia $y$ encopresis, asociados a aislados, en los cuales se pesquisó, mediante estudio urodinámica, vejiga hiperactiva o hipertónica.

Los pacientes con antecedente de infección urinaria recurrente fueron tratados durante el estudio con nitrofurantoína cn dosis de $2 \mathrm{mg} \times \mathrm{kg} x$ día oral, con el objeto de evitar repurrencia de infección urinarja que pudiera alterar el registro urodinúmion, $y$ en consecuencia la interpretación de los resultados.

Los $\mathbf{3 0}$ pacientes fueron evaluados mediante cist ometria retrógradal al inicio del tratamiento farmacológico, $\mathrm{y}$ al tórmino de éste, a los 90 dias. Paralelamente la evaluación clínica se realizó cada 15 días hasta 90 días después de suspendido el fármaco. Para este efecto se confeccionó una ficha tipo, individual, donde se consiynó presencia y frecuencia de infección urinaria, enuresis, incontinencia, encopresis y reacciones adversas al medicanento.

De los 30 pacientes, $5(16,7 \%)$ sran yarones y 25 $(83,3 \%$ eran mujeres. Las edades tluctuaton entre 5 aǹos 2 meses y 15 años 6 meses, con promcdio de 9,8 años. Fil peso promedio fue de $30,9 \mathrm{~kg} \pm 8,9$ D.F. 21 pacicntes (70) tenían vejiga hiperactiva y $9(30 \%)$ vejiga hipertónica. La forma de presentación clinica, en ambas tipos de trastorno vesical, fue similar y se resume on Ja Tabla 1. La capacidad vesical y al residuo postmiccional al injeio del estudio son descritos en la Tabla 2 .

Fl Metil Sulfato de N-Metil Hioscina o N-Metil Escopolamína (UL]X包) es un detivado sintético de la A tropina, on su molécula posee un amonio cuaternario que excluye toda pasibilidad de traspaso a través de la barrera hema toencefálica, y se caracteriza por un efecto anticolinérgico periférico significativo y escasa acción gangliopléjica.

Todos los pacjentes recibieron en la primera semana $3 \mathrm{mg} x$ día por vía oral del medicamento. Posteriomente fueron subdivididos en 3 grupos de acuerdo a su peso. de modo que los niños cuyo peso cra igual o inferior a $20 \mathrm{~kg}$. recibieron $6 \mathrm{mg}$. diarios, los que pesaban 21 a $30 \mathrm{~kg}$. se trataron con $9 \mathrm{mg}$. por día y los que tenían peso de $30 \mathrm{~kg}$. o más, recibieron $12 \mathrm{mg}$. diarios de la droga, hast a completar 90 dias de tratamiento.
Tabla 1.

Presentación clísica de los pacientes con vejiga hipertónica e hiperactiva.

(n: 30)

\begin{tabular}{lr}
\hline Presentación Clínica & $\mathrm{n}$ \\
\hline ENU & 1 \\
ENU + ITL & 3 \\
ENU + INC & 2 \\
ENU + INC + ITU & 12 \\
ENU + INC + ENC & 4 \\
ITU + INC & 1 \\
ITL + INC + FNC & 4 \\
ITU + FNU + INC + ENC & 30 \\
\hline TOTAL
\end{tabular}

$\mathrm{ENC}^{*}=$ Enuresis

ITU = Infección urintaria securrente

INC = Incontinencia urinaria

ENC $=$ Encopresis

\section{RESULTADOS}

Al térnino del tratamiento los 30 pacientes fueron estudiados urodinátnicamente. Los resul. tados del estudio de control se muestran en la Tabla 2.

De los 21 pacientes que en la primera cistometria presentaton vejiga hiperactiva, en 9 (42.9\%) los resultados fueron normales, en 7 $(33,3 \%)$ no se observaron cambios y $5(23,8 \%)$ tuvieron evidencia de vejiga hipotónica. De los 9 pacientes con vejiga hipertónica, en $7(77,8 \%)$ se normalizaron los registros urodinámicos y en 2 $(22,2 \%)$ éstos permanecieron sin cambio.

Los resultados clínicos con respecto a la enuresis, incontinencia y encopresis fueron evaluados según el porcentaje de variación con respecto al intcio del estudio, considerando como mejoría clínica satisfactoria la reducción de por lo menos

Tablin 2.

Capacidad vesical y residuo post miccional en pacientes con vejigas hiperactivas e hipertónjcas en la primera cistometría y segunda cistometría.

(n: 30)

\begin{tabular}{|c|c|c|c|c|}
\hline & \multicolumn{2}{|c|}{ Primera Cistometría } & \multicolumn{2}{|c|}{ Segunda Cistometría } \\
\hline & $\begin{array}{l}\text { Capacidad } \\
\text { Vesical } \\
\text { (ml) }\end{array}$ & $\begin{array}{c}\text { Residuo } \\
\text { post miccional } \\
\text { (ml) }\end{array}$ & $\begin{array}{l}\text { Capacidad } \\
\text { vesicil } \\
\text { (ml) }\end{array}$ & $\begin{array}{c}\text { Residuo } \\
\text { post miccional } \\
\text { (ml) }\end{array}$ \\
\hline $\begin{array}{l}\text { Vejiga } \\
\text { hiperactiva }\end{array}$ & 183,8 DS. 77,8 & 9,5 DS. 6,7 & 222,4 DS. I07,7 & 7,85 DS. 7,16 \\
\hline $\begin{array}{l}\text { Vejiga } \\
\text { Hipertónica }\end{array}$ & 142,2 DS. 61,2 & 12,4 DS. 8,9 & 198,3 DS. 72 & 10,1 DS. 22,5 \\
\hline
\end{tabular}

$P>0,0 S$ 
$75 \%$ de la frecuencia de los signos estudiados y como fracaso el resto.

En la Tabla 3 se observa el resultado clínico de los pacientes con vejiga hiperactiva e hipertónica, at térnino del tratamiento y a 90 días después de suspendido el fármaco.

La correlación clínico-cistométrica muestra que entre aquellos pacientes que inicialmente tenían vejiga hiperactiva y que al término del tratamiento mostraban cistometrías normales, $62,5 \%$ tuvieron mejoría clínica satisfactoria de la enuresis, $57,2 \%$ de la incontinencia, y $66,7 \%$ de la encopresis.

Tabla 3.

Resultado cúnico en 30 niños a los 90 días de tratamiento con ULIX ${ }^{\circledR}$ y a tos 90 días de suspensión del fármaco. Cantidad y percentaje de pacientes con fracaso al tratamiento.

\section{HIPFRACTIVAS}

\begin{tabular}{|c|c|c|c|c|}
\hline \multirow{2}{*}{$\begin{array}{l}\text { Pre } \\
\text { tratamiento } \\
\qquad \text { No }\end{array}$} & \multicolumn{2}{|c|}{$\begin{array}{c}90 \text { días } \\
\text { tratamiento }\end{array}$} & \multicolumn{2}{|c|}{$\begin{array}{l}90 \text { días suspensión } \\
\text { tratamiento }\end{array}$} \\
\hline & yo & $\%$ & $\mathrm{NO}^{\circ}$ & $\%$ \\
\hline $\begin{array}{l}\text { Enutesis } 18 \\
\text { Inconti- }\end{array}$ & 7 & $(38,9)$ & 4 & \\
\hline $\begin{array}{r}\text { nencia } 19 \\
\text { Tencopresis } 6\end{array}$ & $\begin{array}{l}4 \\
1\end{array}$ & $\begin{array}{c}(21) \\
(16,7)\end{array}$ & $\frac{1}{2}$ & $\begin{array}{l}(5,3) \\
(33,3)\end{array}$ \\
\hline
\end{tabular}

\section{HIPERTONICAS}

\begin{tabular}{llcccc}
\hline $\begin{array}{c}\text { Enuresis } \\
\text { Inconti- }\end{array}$ & 8 & 6 & $(75)$ & 3 & $(37,5)$ \\
$\begin{array}{c}\text { nencia } \\
\text { Encopresis }\end{array}$ & 3 & 0 & $(14,2)$ & 0 & $(0)$ \\
\hline
\end{tabular}

En los pacientes cuyas vejigas inicialmente егал hiperactivas y luego resultaron hipotónicas, se obtuvo $66,6 \%$ de mejoria clinica satisfactoria de la enuresis, $80 \%$ de la incontinencia y $100 \%$ de la encopresis. En los casos cuyas vejigas siguieron hiperactivas luego del tratamiento, el 66,6\% mejoró de la enuresis y $100 \%$ de la incontinencia y la encopresis.

Aquellos pacientes que inicialmente presentaron vejiga hipertónica y que al término del tratamiento tenian resultados normales en la cistometría, la enuresis mejoró en $33,3 \%$, la incontinencia en $83,3 \%$ y la encopresis en todos.

En los casos en que las vejigas eran inicialmente hipertónicas y permanecieron sin variación posterior al tratamiento farmacológico, no se registraron mejorías clínicas en la enuresis y sólo en $50 \%$ mejoró la incontinencia.

En la Tabla 4 se expresan los resultados de la correlación clínico cistométrica al término del tratamiento y a los 180 días de seguimiento, es decir, 90 días después de suspendido el medicamento.

Cabe destacar que sólo 5 pacientes $(16,5 \%)$ presentaron algún tipo de reacción adversa al medicamento, que se manifestó por cefalea leve y esporádica, crisis de rubor, mareos y alteraciones visuales transitorias. En ningún caso fue necesaria la suspensión o disminución de la dosis del fármaco, ya que los sintomas cedieron en forma espontánea.

\section{DISCUSION}

Luego de 90 días de tratamiento con sulfato $\mathrm{N}$-Metil hoscina se observó un incremento del $25,5 \%$ de la capacidad vesical promedio en niños con vejiga hiperactiva e hipertónica. Sin embargo, la diferencia entre las medias de las capacidades vesicales pre y post tratamiento no tiene valor estadísticamente significativo $(p>0,05)$. Asimismo observamos una disminución promedio del $10 \%$ en la amplitud del segmento de reflexia en las vejigas hipertónicas y la disminución o desaparición de las contracciones no inhibidas en las vejigas hiperactivas. Esto explicaría el efecto

Tabla 4.

Correlación urodinámica-clínica a los 90 días de tratamiento con ULIX@y a los 90 dias de suspendido el fármaco.

\begin{tabular}{|c|c|c|c|c|c|c|c|}
\hline \multicolumn{2}{|c|}{$\begin{array}{l}\text { Resultado } \\
\text { Urodinámico }\end{array}$} & \multicolumn{6}{|c|}{$\begin{array}{l}\text { Resultado clínico en porcentaje de } \\
\text { pacientes } x \text { n respuesta satisfactoria* }\end{array}$} \\
\hline \multirow{2}{*}{$\begin{array}{l}\text { 1ra. cistometría } \\
\text { (iniciab) }\end{array}$} & \multirow{2}{*}{$\begin{array}{l}\text { 2a. cistometría } \\
\text { (90 dias) }\end{array}$} & \multicolumn{2}{|c|}{ Enuresis } & \multicolumn{2}{|c|}{ Incontinencia } & \multicolumn{2}{|c|}{ Enoopresis } \\
\hline & & $90 \mathrm{ds}$. & $180 \mathrm{ds}$ & $90 \mathrm{ds}$ & $180 \mathrm{ds}$ & $90 \mathrm{ds}$ & $180 \mathrm{ds}$ \\
\hline Hiperactivas & $\begin{array}{l}\text { Normales } \\
\text { Hiperactivas } \\
\text { Hipotónicas }\end{array}$ & $\begin{array}{l}62,5 \\
66,6 \\
66,6\end{array}$ & $\begin{array}{l}75 \\
85,7 \\
100\end{array}$ & $\begin{array}{l}57,2 \\
100 \\
80\end{array}$ & $\begin{array}{l}100 \\
87,5 \\
100\end{array}$ & $\begin{array}{l}66,7 \\
100 \\
100\end{array}$ & $\begin{array}{l}66,7 \\
100 \\
100\end{array}$ \\
\hline Häpertónicas & $\begin{array}{l}\text { Normates } \\
\text { Hipertónicas }\end{array}$ & $\begin{array}{c}33,3 \\
0\end{array}$ & $\begin{array}{l}83,3 \\
0\end{array}$ & $\begin{array}{l}83,3 \\
50\end{array}$ & $\begin{array}{l}100 \\
100\end{array}$ & $\begin{array}{r}100 \\
-\end{array}$ & $\begin{array}{r}100 \\
-\end{array}$ \\
\hline
\end{tabular}

\footnotetext{
* Reducción de a lo menos et $75 \%$ de la frecuencia de lo signos consignados.
} 
clinico favorable en ambos tipos de trastornos vesicales después del tratamiento.

Más de $60 \%$ de los pacientes que al inicio del estudio presentaron vejiga hiperactiva obtuvieron respuesta clínica satisfactoria en los 3 signos evaluados, especialmente en la incontinencia y la encopresis.

La evaluación clinica efectuada 90 dias des. pués de suspendido el medicamento permitió constatar que la respuesta no sólo se mantuvo sino que mostró una mejoría.

Este fenómeno no se explica por la farmacocinética del medicamento, ya que su vida media es menor de 24 horas.

Del grupo de niños que inicialmente presentó vejigas hipertónicas, más del $80 \%$ obtuvo una respuesta clínica satisfactoria con respecto a la incontinencia y la encopresis. En cambio, sólo e] $30 \%$ de los pacientes obtuvo respuesta clínica similar sobre la enuresis.

La evolución clínica a los 90 días de suspendida la droga muestra una tendencia a la mejoría, cuya interpretación no es confiable debido al escaso número de pacientes que constituye este grupo.

La normalización del registro urodinámico no indica necesariamente mejoria clínica y viceversa, ya que aquellos enfermos que normalizaron su registro cistométrico no difirieron en el resultado clínico de aquellos que permanecieron urodinámicamente hiperactivos.

De los pacientes que inicialmente presentaron vejiga hiperactiva, 23,8\% mostraron al término de la terapia un registro urodinámico hipotónico. La totalidad de ellos mejoró clínicamente en cuanto a la enuresis, incontinencia y encopresis. Los registros cistométricos hipotónicos no nos parecen debidos a sobredosis de medicamento, pues sólo un niño presentó crisis de rubor transitoria que cedió espontáneamente.

Fínalmente nos permitimos recomendar el ușo de sulfato de N-Metil hioscina en niños con vejigas hiperactivas o hipertónicas que presentan enuresis, incontinencia o cncopresis, en forma aislada o asociada, en la dosis señalada en el presente trabajo.

Los resultados a largo plazo no son predecibles de acuerdo a la experiencia presentada.

\section{RESUMEN}

Se presentan 21 niños con vejiga hiperactiva y
9 con yejiga hipertóntca que consultaron por enuresis, incontinencia o encopresis en forma aislada o asociada y que recibieron durante 90 dias tratamiento con sulfato de $\mathrm{N}$-Metil Hioscina. Todos aquellos pacientes que presen. taban infección urinaria recurrente se trataron durante todo el estudio con Nitrofurantoina $2 \mathrm{mg}$. $\mathrm{x} \mathrm{kg/díi.} \mathrm{En} \mathrm{todos} \mathrm{se} \mathrm{efectuó} \mathrm{registro}$ urodinamico al inicio $\mathrm{y}$ al término del tratamien. to farmacológico. El estudio clínico se prolongó durante 90 días de suspendido el tratamiento.

Se comprobó un aumento no significativo $(\mathrm{P}>0.05)$ en la capacidad vesical y en el residuo promedio, una menor amplitud del segmento de reflexia $y$ desaparición o disminución de las contracciones no inhibidas en las vejigas hiperactivas, al término de la terapia.

Se observó una respuesta clínica satisfactoria en los pacientes con vejigas hiperactivas independiente del registro urodinámico al término del tratamiento sobre incontinencia, enuresis y encopresis, resultado que se prolongó hasta los 90 dias de seguimiento. En los pacientes con vejiga hipertónica, la respuesta clínica fue satisfactoria sobre la incontinencia y la encopresis y mala sobre la enuresis. Esta respuesta se matuvo durante todo el tiempo de observación. No se apreció efectos colaterales indeseables por acción del medicamento.

\section{REFERENCLAS}

1. Rodrígucz E., Holzer J., Triviño $x . y$ Col.: La vejiga inestable en la infancia. Rev. Chil. Pcdiatr. 56: 45 , 1985 .

2. Rodriguez E., Holzer J., Triviño X. y Col.: Capacidad vesical, edad, peso, talla y superficie corporal en niños. Rev. Chil. Pediatr. 56: 81, 1985.

3. Wolker D.: Pharmacologic and surgical mamagement of unstable bladder. Dialogues in Pediatric Urology 9: 1, 1984 .

4. Wein A.: Pharmacologic management of unstable bladder: Dialogues in Pediatric Urology 9: 4, 1984

5. Kroovand R., Perlmutter A.i Neurogenic Bladder. Pediatric Surgery Ed. Saunders Company, Págs. $664-665,1980$.

6. Staskin $D$., Parsons $K$. et al: $\mathbf{B}$ ladder transection - a functional, neurophysiological, neuropharmacological and neuroanatomical study. Br. J. Urol. 53: $552,1981$.

7. Kao Hwang: Fharmacological background of modern anticholinergic drugs. Am. J. Gastroenterol. 26: 56, 1956.

8. Anonimo. Methyl sulfate of N-Methyl Hyoschine: Compendio Famacológico Laboratorio Andrómaco, 1984. 